\title{
Personalized Treatment of Advanced Gastric Cancer Guided by the MiniPDX Model
}

\author{
Jianzheng Wang, ${ }^{1}$ Jinxi Huang, ${ }^{2}$ Hui Wang, ${ }^{3}$ Wei Yang, ${ }^{2}$ Qiwen Bai, ${ }^{3}$ Zhentao Yao, \\ Qingli Li, ${ }^{4}$ Huifang Lv, ${ }^{1}$ Beibei Chen, ${ }^{1}$ Caiyun Nie, ${ }^{1}$ Weifeng Xu, ${ }^{1}$ Shuiping Tu $\mathbb{D},{ }^{4}$ \\ Hongle $\mathrm{Li}^{5}$ and Xiaobing Chen $\mathbb{D}^{1}$ \\ ${ }^{1}$ Department of Medical Oncology, Affiliated Cancer Hospital of Zhengzhou University, Henan Cancer Hospital, Zhengzhou, \\ Henan Province 450008, China \\ ${ }^{2}$ Department of General Surgery, Affiliated Cancer Hospital of Zhengzhou University, Henan Cancer Hospital, Zhengzhou, \\ Henan Province 450008, China \\ ${ }^{3}$ Department of Endoscope Center, Affiliated Cancer Hospital of Zhengzhou University, Henan Cancer Hospital, Zhengzhou, \\ Henan Province 450008, China \\ ${ }^{4}$ Department of Oncology, Renji Hospital, School of Medicine, Shanghai Jiaotong University, Shanghai 200127, China \\ ${ }^{5}$ Department of Molecular Pathology, Affiliated Cancer Hospital of Zhengzhou University, Henan Cancer Hospital, Zhengzhou, \\ Henan Province 450008, China
}

Correspondence should be addressed to Shuiping Tu; tushuiping@yahoo.com and Xiaobing Chen; zlyychenxb0807@zzu.edu.cn

Received 3 December 2021; Accepted 3 January 2022; Published 27 January 2022

Academic Editor: Dong-Hua Yang

Copyright (c) 2022 Jianzheng Wang et al. This is an open access article distributed under the Creative Commons Attribution License, which permits unrestricted use, distribution, and reproduction in any medium, provided the original work is properly cited.

\begin{abstract}
Background. The morbidity and mortality of gastric cancer are high in China. There are challenges to develop precise and individualized drug regimens for patients with gastric cancer after a standard treatment. Choosing the most appropriate anticancer drug after a patient developing drug resistance is very important to improve the patient's prognosis. MiniPDX has been widely used as a new and reliable preclinical research model to predict the sensitivity of anticancer drugs. Methods. The OncoVee ${ }^{\circledR}$ MiniPDX system developed by Shanghai LIDE Biotech Co., Ltd. was used to establish the MiniPDX models using specimens of patients with gastric cancer. The cancer tissues were biopsied under endoscopy, and then, the tumor cell suspension was prepared for a drug sensitivity test by subcutaneously implanting into Balb/c-nude mice. The selected optimal regimen obtained from the MiniPDX assay was used to treat patients with drug-resistant gastric cancer. Results. We successfully established an individualized and sensitive drug screening system for four patients from January 2021 to July 2021. MiniPDX models identified potentially effective drugs for these four patients, with partial remission in two of the patients after treatment and disease progression in the remaining of two patients. Severe side effects from chemotherapy or targeted therapy were not observed in all patients. Conclusion. Establishing a personalized drug screening system for patients with drug-resistant gastric cancer can guide the selection of clinical drugs, improve the clinical benefit of patients, and avoid ineffective treatments. It can be an effective supplement for treatment options.
\end{abstract}

\section{Introduction}

Gastric cancer is one of the most common malignancies worldwide and the fourth leading cause of cancer-related death [1]. The disease generally carries a dismal prognosis due to its advanced stage at initial diagnosis. The median survival rate is less than 12 months for patients at the advanced stage [2]. At present, the first-line treatment of metastatic gastric cancer is based on conventional chemotherapy, and there is an urgent need to explore a more effective treatment plan [3]. Except for the positive results of trastuzumab in HER2-positive advanced gastric cancer, other clinical studies on targeted drugs for the treatment of advanced gastric cancer ended in failure [4-6]. In recent 
years, immune checkpoint inhibitors have emerged for advanced gastric cancer with their unique mechanism of action. For HER2-negative gastric cancer, the two major clinical studies of CheckMate649 and Attraction04 have established the clinical utility of nivolumab for the first-line treatment [7, 8]. For HER2-positive gastric cancer, the practice-changing findings of the KEYNOTE-811 trial led the US FDA to grant accelerated approval of pembrolizumab in combination with trastuzumab and fluoropyrimidine and platinum-based chemotherapy as first-line therapy for patients with HER2-positive advanced gastroesophageal junction adenocarcinoma [9]. Therefore, in general, we have standard treatment options for the first-line treatment of advanced gastric cancer.

However, after the first-line or second-line treatment for patients with advanced gastric cancer, how to choose a personalized regimen with accuracy and effectiveness for each patient is currently a great challenge. Although there are many recommendations in the National Comprehensive Cancer Network (NCCN) guidelines or Chinese Society of Clinical Oncology (CSCO) guidelines, there is no final conclusion on which option is the most effective one $[3,10]$. Although next-generation sequencing (NGS) and other genetic tests can be performed after disease progression, many patients cannot find suitable targets or suitable drugs from the test results. Therefore, it is essential to select effective drugs and develop a personalized treatment regimen.

In recent years, more and more researchers have tried to screen anticancer drugs in vitro by simulating tumor growth in vivo. Patient-derived xenograft (PDX) has emerged in this context and has achieved certain clinical results. It is currently the most representative animal model with human tumor genetic information [11-13]. The accuracy of PDX samples on drug efficacy and resistance rates can reach $90 \%$ based on a previous study [14]. Although the PDX drug sensitivity test has obvious advantages, it takes about half a year to go through the traditional PDX drug sensitivity test (the process of modeling, passage, amplification, and efficacy analysis) [12]. The survival time of patients with advanced gastric cancer is short, and the disease progression is rapid [15-17]. Due to the long construction time, the traditional PDX model cannot quickly reflect the drug sensitivity of patients and cannot meet the clinical needs. The latest rapid, human-derived xenograft tumor drug sensitivity detection technology (MiniPDX) solves this problem. These models generate drug sensitivity test results within 7-10 days [18]. Clinical studies have shown that MiniPDX can help improve the prognosis of patients with gallbladder cancer [19].

Therefore, in this study, MiniPDX was used to test the specimens of gastric cancer patients who had undergone pretreatment and select the best anticancer drugs for the subsequent treatment. Based on the MiniPDX assay results, we chose the most effective tumor-inhibiting regimen to evaluate whether it can effectively inhibit tumor growth in patients.

\section{Materials and Methods}

2.1. Collection of Patient Information. We collected a range of baseline information from each patient, including age, gender, medical history, tumor staging, pathological types, Lauren's classification, HER2 status, imaging examination results, and metastatic sites (Table 1).

2.2. Tissue Specimen Acquisition. We performed painless gastroscopy for tissue biopsy in patients with gastric cancer who have progressed after the first-line or second-line treatment under general anesthesia. In addition to routine pathological examination, we harvested enough tissue specimens for quality control before the establishment of MiniPDX models.

2.3. MiniPDX Model Establishment. MiniPDX assay was carried out using the OncoVee ${ }^{\circledR}$ MiniPDX kit (LIDE Biotech Co., Ltd., Shanghai, China) (Figure 1). Gastric cancer samples were harvested and then washed with Hanks' balanced salt solution (HBSS) to remove nontumor and necrotic tumor tissues in a biosafety cabinet. Subsequently, the tumor tissues were digested with collagenase at $37^{\circ} \mathrm{C}$ for 1-2 h. Gastric cancer cells were collected followed by removal of blood cells and fibroblasts. Then, gastric cancer cell suspension was transferred to the HBSS washed OncoVee ${ }^{\circledR}$ capsules. Capsules were implanted subcutaneously via a small skin incision with three capsules per mouse (5-weekold nu/nu mouse), two mice per drug regimen [18]. One day after tumor cell inoculation, tumor-bearing mice were randomly allocated to vehicle group and drug treatment group. We chose 4 to 6 chemotherapy regimens for MiniPDX drug sensitivity tests according to the following principles: frequently used second-line and late-line chemotherapeutic plans and targeted plans for gastric cancer (such as docetaxel, irinotecan, nab-paclitaxel, S-1, and apatinib) were commonly considered; potentially effective molecular-targeted drugs such as pyrotinib and fruquintinib were also selected. Drugs that might cause severe side effects, as indicated by medical history and genetic testing results, were avoided. Drug sensitivity tests were carried out using MiniPDX models, as described later. Finally, we formulated a personalized therapeutic regimen for each patient according to the drug sensitivity test results.

2.4. Drug Sensitivity Test. Mice-bearing MiniPDX capsules were treated with single drugs or combination of drugs as detailed in Table 2 for 7 days. Thereafter, the implanted capsules were removed, and tumor cell proliferation was evaluated using the CellTiter Glo Luminescent Cell Viability Assay Kit, as instructed by the manufacturer. Luminescence was measured in terms of relative luminance unit (RLU) using a spectrophotometer. Relative proliferation rate (RPR) was calculated using the following equation: (mean RLU of the treatment group on day $7-$ mean RLU on day 0$) /($ mean RLU of the vehicle group on day $7-$ mean RLU on day 0$) \times$ $100 \%$. Each experiment was conducted in sextuplicate, and the mean values were reported. A positive drug response was considered if $\mathrm{RPR} \leq 55 \%$, and a negative drug response was considered if RPR $>55 \%$ [20]. 


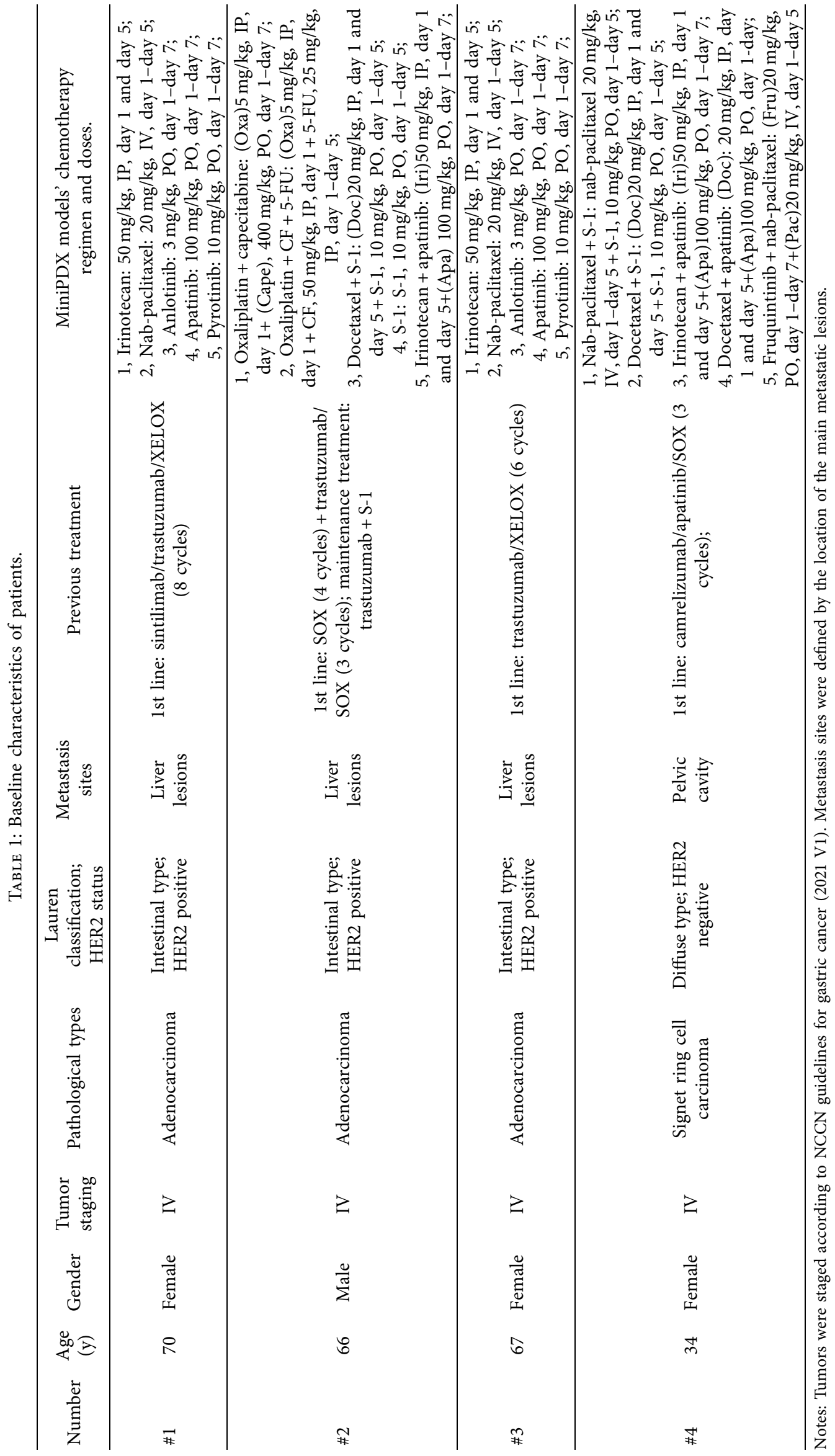



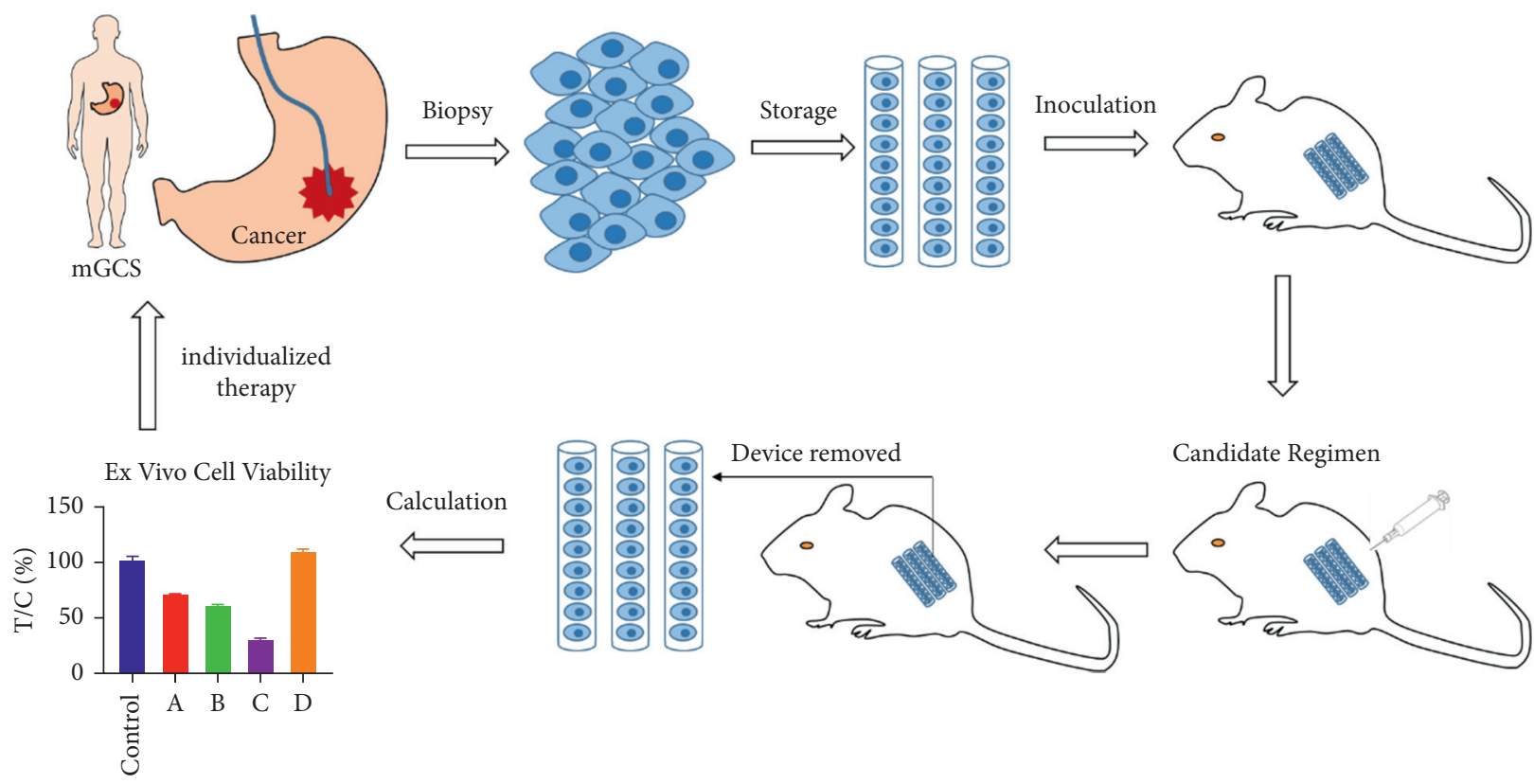

Figure 1: General schema of MiniPDX models of gastric cancer.

TABle 2: MiniPDX drug sensitivity results of the 4 patients.

\begin{tabular}{|c|c|c|c|c|}
\hline Number & Chemotherapy regimen & $\begin{array}{c}\text { Inhibition rate } \\
(1-\mathrm{T} / \mathrm{C} \%)\end{array}$ & Weight loss of mice $>15 \%$ & Source of cancer cells \\
\hline \multirow{5}{*}{ Patient \#1 } & 5, Irinotecan & 68 & - & \multirow{5}{*}{ Gastroscopic biopsy of gastric cancer } \\
\hline & 6, Nab-paclitaxel & 47 & - & \\
\hline & 7, Anlotinib & 46 & - & \\
\hline & 8, Apatinib & 52 & - & \\
\hline & 9, Pyrotinib & 55 & - & \\
\hline \multirow{5}{*}{ Patient \#2 } & 5, Oxaliplatin + capecitabine & 20 & - & \multirow{5}{*}{ Gastroscopic biopsy of gastric cancer } \\
\hline & 6 , Oxaliplatin $+\mathrm{CF}+5-\mathrm{FU}$ & 16 & - & \\
\hline & 7, Docetaxel + S-1 & 6 & - & \\
\hline & $8, \mathrm{~S}-1$ & 23 & - & \\
\hline & 9, Apatinib + irinotecan & 28 & - & \\
\hline \multirow{5}{*}{ Patient \#3 } & 5 , Irinotecan & 80 & - & \multirow{5}{*}{ Gastroscopic biopsy of gastric cancer } \\
\hline & 6, Nab-paclitaxel & $>100 \%$ & - & \\
\hline & 7, Anlotinib & $>100 \%$ & - & \\
\hline & 8, Apatinib & 81 & - & \\
\hline & 5, Pyrotinib & $>100 \%$ & - & \\
\hline \multirow{5}{*}{ Patient \#4 } & 5, Nab-paclitaxel + S-1 & $>100 \%$ & - & \multirow{5}{*}{ Gastroscopic biopsy of gastric cancer } \\
\hline & 6, Docetaxel + S-1 & $>100 \%$ & - & \\
\hline & 7, Irinotecan + apatinib & $>100 \%$ & - & \\
\hline & 8, Docetaxel + apatinib & 66 & - & \\
\hline & 9, Fruquintinib + nab-paclitaxel & 71 & - & \\
\hline
\end{tabular}

1, Notes: Inhibition rate was calculated by $1-\mathrm{T} / \mathrm{C} \%(\mathrm{~T} / \mathrm{C} \%=$ treatment group proliferation rate/control group proliferation rate\%). Each regimen was used on MiniPDX models with the same doses. Detailed doses for the MiniPDX models-irinotecan: $50 \mathrm{mg} / \mathrm{kg}$, IP, day 1 and day $5 ;$ nab-paclitaxel, $20 \mathrm{mg} / \mathrm{kg}$, IV, day 1-day 5; anlotinib, $3 \mathrm{mg} / \mathrm{kg}$, PO, day 1-day 7; apatinib: $100 \mathrm{mg} / \mathrm{kg}$, PO, day 1-day 7; pyrotinib, $10 \mathrm{mg} / \mathrm{kg}$, PO, day 1-day 7; (oxaliplatin + capecitabine): oxaliplatin (Oxa), $5 \mathrm{mg} / \mathrm{kg}$, IP, day $1+$ capecitabine (Cape), $400 \mathrm{mg} / \mathrm{kg}$, PO, day 1-day 7; (oxaliplatin + CF+5-FU): oxaliplatin, $5 \mathrm{mg} / \mathrm{kg}, \mathrm{IP}$, day $1+\mathrm{CF}, 50 \mathrm{mg} /$ $\mathrm{kg}$, IP, day 1 + 5-FU, $25 \mathrm{mg} / \mathrm{kg}$, IP, day 1-day 5; (docetaxel + S-1): docetaxel (Doc): $20 \mathrm{mg} / \mathrm{kg}$, IP, day 1 and day 5 + S-1, $10 \mathrm{mg} / \mathrm{kg}$, PO, day 1-day 5; S-1: S-1, $10 \mathrm{mg} / \mathrm{kg}$, PO, day 1-day 5; (irinotecan + apatinib): $50 \mathrm{mg} / \mathrm{kg}$, IP, day 1 and day 5+ $100 \mathrm{mg} / \mathrm{kg}$, PO, day 1-day 7; (nab-paclitaxel + S-1): nab-paclitaxel $20 \mathrm{mg} /$ $\mathrm{kg}$, IV, day 1-day $5+\mathrm{S}-1,10 \mathrm{mg} / \mathrm{kg}$, PO, day 1-day 5; (docetaxel + apatinib): docetaxel: $20 \mathrm{mg} / \mathrm{kg}$, IP, day 1 and day 5 + apatinib: $100 \mathrm{mg} / \mathrm{kg}$, PO, day 1-day 7; (fruquintinib + nab-paclitaxel): fruquintinib (Fru): $20 \mathrm{mg} / \mathrm{kg}$, PO, day 1-day $7+20 \mathrm{mg} / \mathrm{kg}$, IV, day 1-day 5 .

2.5. The Prediction of Side Effects. The potential side effects of each protocol were investigated by determining the weight loss of mice in the MiniPDX system. Weight loss was recorded as RCBW\% (rate of change in body weight) and was calculated as follows: $\mathrm{RCBW} \%=(\mathrm{BWi}-\mathrm{BW} 0) / \mathrm{BW} 0 * 100 \%$, in which BWi represented the body weight of the mouse on day 1 and BW0 represented the body weight of the mouse when the MiniPDX model began. Each regimen was tested in 
two mice, and final RCBW\% was calculated as the average number of the two repeats; $15 \%$ was chosen as a cutoff point to predict whether we would consider avoiding this regimen due to the possibility of severe side effects [21].

2.6. Statistical Analysis. Statistical data and graphics were analyzed using GraphPad Prism 8. Data are presented as mean \pm standard deviation (SD). Statistical significance was assessed by Student's $t$ test, with $P<0.05$ indicating significance.

\section{Results}

3.1. Basic Clinical Information. From January 2021 to July 2021, we constructed and tested the MiniPDX models in four patients with advanced gastric cancer. Before constructing the mouse models, we conducted in-depth communication with patients and their families, and each patient signed an informed consent form. The four patients with advanced gastric cancer have progressed after previous treatment, and there was no precise and effective plan to guide their subsequent treatment. To avoid ineffective attempts, we took biopsies of primary gastric cancer in these four patients under endoscopy and then tested the drug sensitivity by establishing the MiniPDX models (Table 1). Patient \#1 suffered from HER2positive gastric cancer with liver metastases. Sintilimab combined with XELOX (L-OHP combined with capecitabine) was used as the first-line treatment. After treatment, the primary gastric cancer progressed, but the liver metastases shrank. Patient \#2 had HER2-positive gastric cancer with liver metastasis. The first-line treatment was SOX $(\mathrm{L}-\mathrm{OHP}+\mathrm{S}-1)$ regimen for four cycles, followed by trastuzumab combined with SOX regimen for three cycles. Then, trastuzumab combined with S-1 was used for maintenance therapy. During the treatment, both primary gastric cancer and liver metastases have progressed. Patient \#3 also had HER2-positive gastric cancer with liver metastases. The first-line treatment was trastuzumab combined with XELOX for 6 cycles. The primary gastric cancer progressed, and the liver metastases shrank. Patient \#4 was diagnosed with diffuse gastric cancer with pelvic metastasis, and the pathological type was signet ring cell carcinoma. The first-line treatment was 3 cycles of camrelizumab, apatinib, and SOX. The primary gastric cancer and the pelvic metastases were significantly enlarged. Biopsies of all primary gastric tumors and metastases were performed in these four patients. However, no cancer cells were found in the liver metastases of patient \#1 and patient \#3, which might be related to the necrotic lesion after chemotherapy or targeted therapy. MiniPDX models failed to be established from the liver metastases of patient $\# 2$ and the pelvic metastases of patient \#4 because tissues were too little to be punctured. In the end, the MiniPDX models were successfully established from the biopsy tissues of these four patients with primary gastric cancer lesions.

3.2. MiniPDX Drug Sensitivity Results. A regimen was defined as "sensitive" when the proliferation rate of the tumor cells was under $55 \%$ compared to the control group [21]. The subsequent choice of the chemotherapeutic regimen was guided by the drug sensitivity test results. According to the test results of MiniPDX and the CSCO guidelines, we selected the most suitable regimen for treatment for each patient. Patient \#1 received nab-paclitaxel as second-line treatment. Although the MiniPDX results showed that the inhibitory effect of anlotinib was slightly more potent than that of nab-paclitaxel, there is more evidence-based medicine for albumin paclitaxel as second-line treatment [22]. Patient \#2 was treated with docetaxel combined with S-1 as second-line treatment because it has the most potent tumor inhibitory effect among the five chemotherapy regimens selected. It was challenging to choose the treatment plan for patient \#3 and patient \#4 because the MiniPDX models showed resistance to all types of chemotherapeutic and targeted drug candidates. The patient \#3 was treated with apatinib, and docetaxel combined with apatinib was used for patient \#4. Both patients and their families were informed that the treatment effects might not be sound (Figure 2).

3.3. Side Effect Predictions and Measurements. Patients with advanced gastric cancer often have malnutrition and poor physical status. Therefore, plans chosen for each patient should consider not only the efficacy, but also the adverse reactions and toxicity. MiniPDX is the model that can predict not only a curative effect but also the toxicity. It is possible that the regimen would cause serious side effects if the mouse suffered a loss in body weight that exceeded 15\% during the 7-day treatment. Alternatives were considered when the inhibitory rate was similar. The degree of weight loss for each mouse model is shown in Figure 3. We found that the nude mice paired with patients \#1, patient \#2, patient \#3, and patient \#4 did not show significant weight loss. Afterward, the patients did not show significant toxic and side effects after adopting the selective treatment plan. Mild side effects, according to the CTCAEv5.0 standards, are described in Table 3.

3.4. Treatment Outcomes. All 4 patients used relatively sensitive regimens based on the predicted results of the MiniPDX models. Following treatment, patient \#1 and patient \#2 reached partial response (PR) according to the Response Evaluation Criteria in Solid Tumors (RECIST) Version1.1 guidelines. However, patient \#3 and patient \#4 showed disease progression (Figure 4). The detailed treatment plans that were developed after drug selection are shown in Table 3. Specifically, patient \#1 and patient \#4 received sintilimab and camrelizumab, respectively, in the first-line treatment. Although the clinical data of immunotherapy in crossline treatment are few, the combination of immunotherapy with selected drugs was administered due to previous treatments with donated drugs.

\section{Discussion}

Patients with advanced gastric cancer have a short survival time and a poor overall prognosis. The median overall survival of patients with advanced disease (locally advanced 

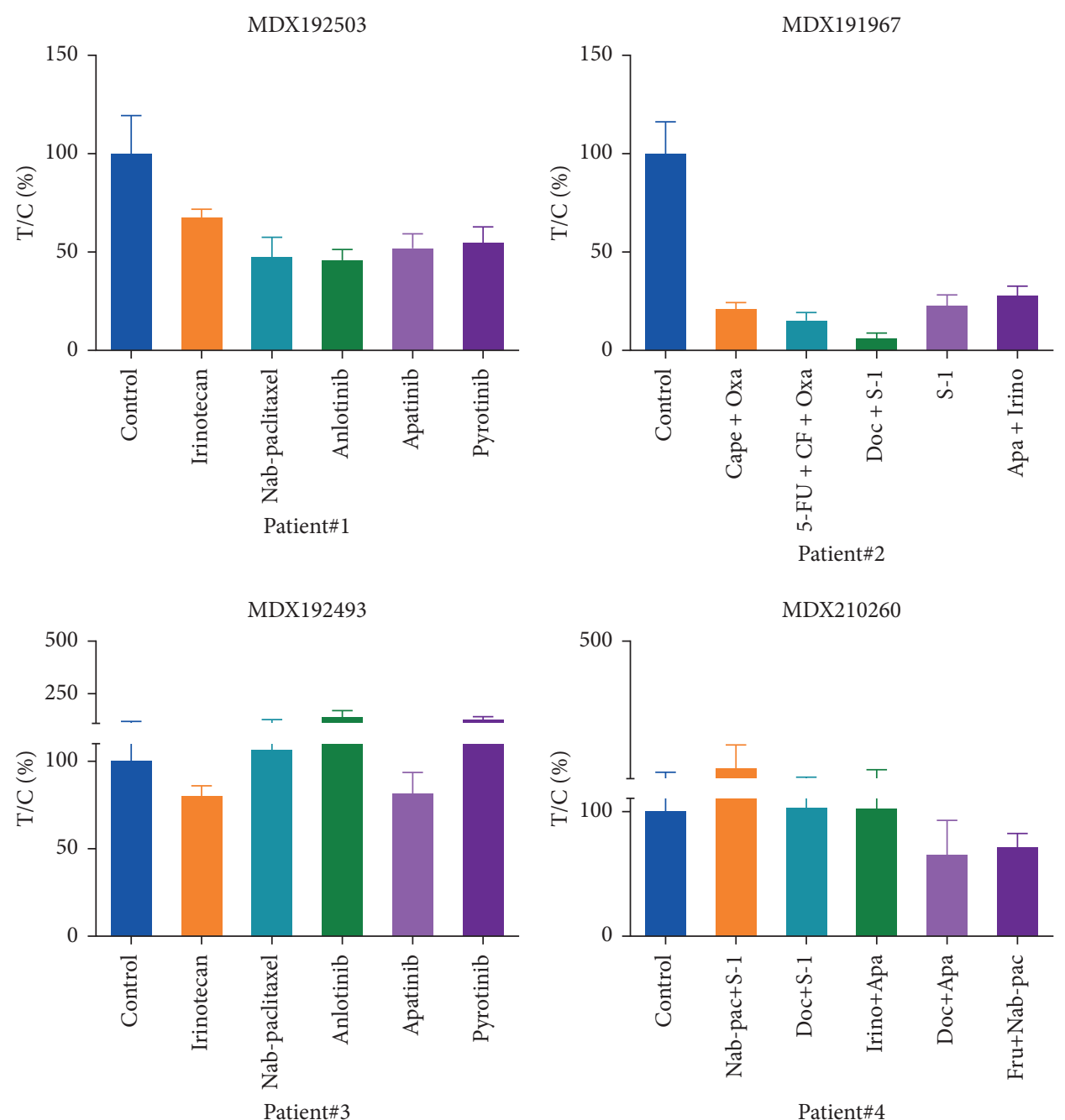

Figure 2: Drug selection test results for the MiniPDX models based on the four patients. Tumor cell growth $(\mathrm{T} / \mathrm{C} \%=$ treatment group proliferation rate/control group proliferation rate\%) was calculated using the formula: (mean RLU of the treatment group on day 7-mean RLU on day 0)/(mean RLU of the vehicle group on day 7-mean RLU on day 0). Abbreviations for chemotherapy: Nab-pac (nab-paclitaxel); Cape (capecitabine); Oxa (oxaliplatin); Irino (irinotecan); Apa (apatinib); Doc (docetaxel); Fru (fruquintinib); 5-FU (5-fluorouracil); CF (calcium folinate).

or metastatic) was 10-12 months. [23]. Although various gastric cancer guidelines provide us with standardized diagnoses and treatment plans, due to individual differences and tumor heterogeneity [24], a certain plan is effective for one patient but ineffective in another. Therefore, a more precise plan to provide individualized treatment for each patient is urgently needed.

With the advent of the increasing number of targeted drugs and new chemotherapeutics, choosing individualized drugs for patients has become a challenge for clinicians. The PDX model could conserve the tumor microenvironment of the primary tumor. Compared to the previous cell-derived xenograft (CDX), PDX reserves the pathophysiology, histology, and phenotypic characteristics of primary tumors. The drug sensitivity test has a high consistency with clinical application, which is crucial in precise tumor treatment $[25,26]$. Coclinical trials were run in parallel with human clinical trials in real time, and mouse trials using PDX models established from participants of clinical trials to evaluate drug response. This method is recognized as a model for personalized treatment or precision medicine $[27,28]$. The PDX model used in coclinical trials is also called as "avatar" or "mirror" model. Some studies have reported that the patients and their PDX models had a high agreement rate of drug response $[29,30]$, indicating that these models can function as "mirror" models for donor patients. In addition, a PDX model can be treated not only with the same drug used in the donor patients but also with other drugs or a novel drug combination. The PDX model, in this case, an "avatar" model, can predict both the development of resistance to first-line therapy and the response to second-line therapy before these events are observed in the donor patient [31].

The advantage of the MiniPDX model is that we can quickly obtain the results of drug sensitivity experiments that are highly consistent with the PDX test results. 
MDX192503

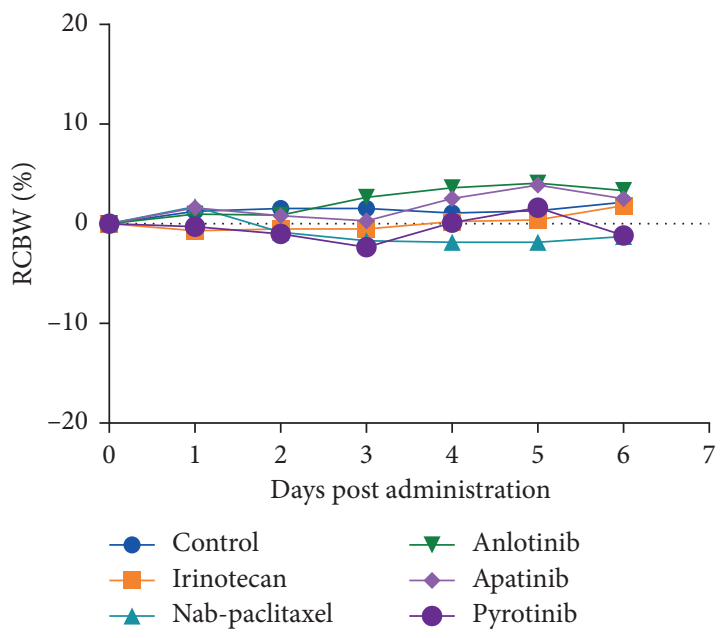

Patient\#1

MDX192493

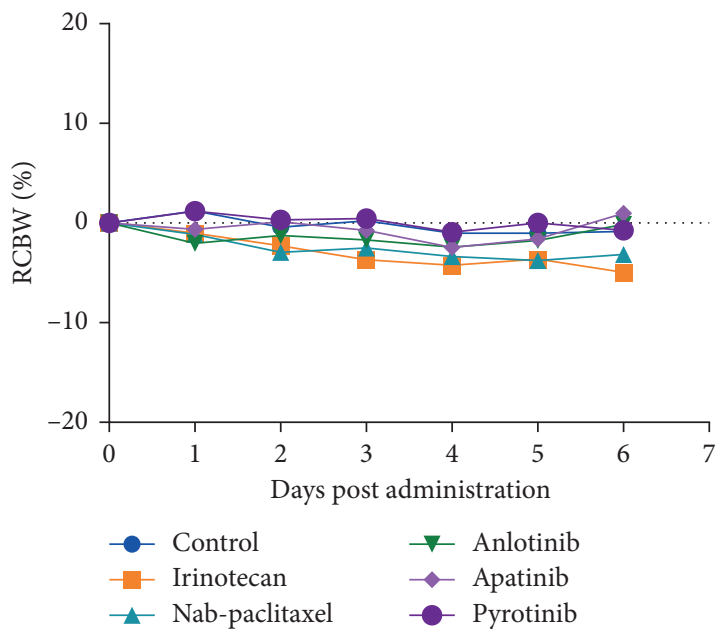

Patient\#3

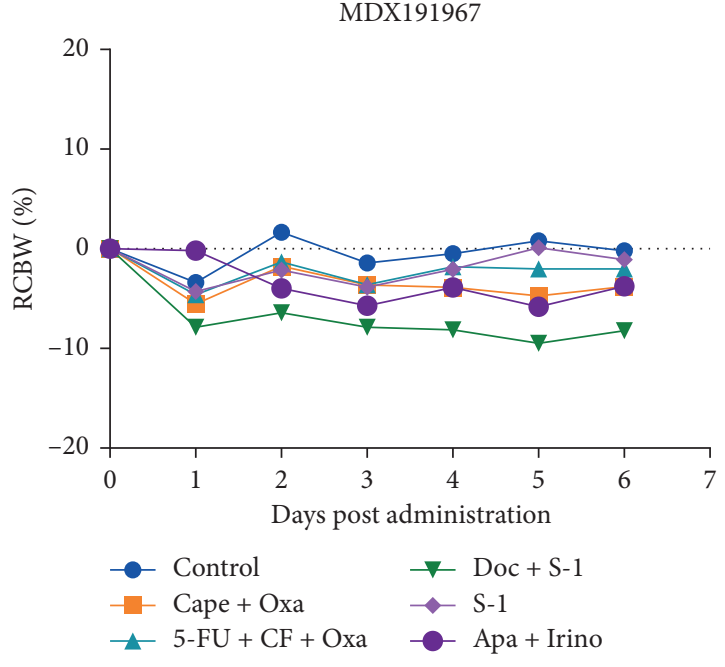

Patient\#2

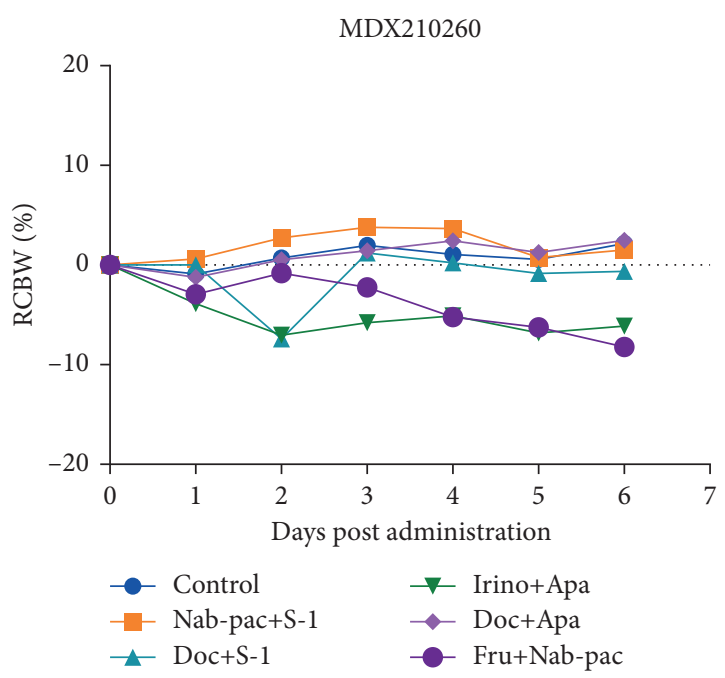

Patient\#4

Figure 3: The loss of weight in mice during the 7-day drug treatment. RCBW\% $=(\mathrm{BWi}-\mathrm{BW} 0) / \mathrm{BW} 0 * 100 \%$; $\mathrm{BWi}$ represents the body weight of the mice on day 1, while BW0 represents the body weight of mice when the MiniPDX model was first established. Abbreviations for chemotherapy: Nab-pac (nab-paclitaxel); Cape (capecitabine); Oxa (oxaliplatin); Irino (irinotecan); Apa (apatinib); doc (docetaxel); Fru (fruquintinib); 5-FU (5-fluorouracil); CF (calcium folinate).

TABLE 3: Treatment after enrollment, clinical response, and side effects.

\begin{tabular}{|c|c|c|c|}
\hline $\begin{array}{l}\text { Patient } \\
\text { number }\end{array}$ & Treatment regimen after drug selection & $\begin{array}{l}\text { Clinical } \\
\text { outcome }\end{array}$ & Side effects \\
\hline$\# 1$ & Sintilimab: $200 \mathrm{mg} \mathrm{d} 1$, nab-paclitaxel: $180 \mathrm{mg} \mathrm{d} 1,8, \mathrm{q} 21 \mathrm{~d} * 2$ cycles & $\mathrm{PR}$ & $\begin{array}{c}\text { Leukopenia (grade II) } \\
\text { Thrombocytopenia } \\
\text { (grade II) } \\
\text { Alopecia (grade I) }\end{array}$ \\
\hline$\# 2$ & Docetaxel: $60 \mathrm{mg} \mathrm{d} 1,8, \mathrm{~S}-1: 60 \mathrm{mg}$ PO bid d1-14, q21d $* 2$ cycles & $\mathrm{PR}$ & $\begin{array}{l}\text { Alopecia (grade I) } \\
\text { Leukopenia (grade I) }\end{array}$ \\
\hline$\# 3$ & Apatinib: $250 \mathrm{mg}$ PO qd $* 2$ cycles & $\mathrm{PD}$ & $\begin{array}{l}\text { Nausea (grade II) } \\
\text { Anemia (grade II) }\end{array}$ \\
\hline$\# 4$ & $\begin{array}{l}\text { Camrelizumab: } 200 \mathrm{mg} \text { d1 } \mathrm{q} 14 \mathrm{~d} \text {, docetaxel: } 60 \mathrm{mg} \text { d1, 8, apatinib: } 250 \mathrm{mg} \text { PO } \\
\text { qd } * 1 \text { cycle, } \mathrm{q} 21 \mathrm{~d}\end{array}$ & $\mathrm{PD}$ & $\begin{array}{l}\text { Nausea (grade II) } \\
\text { Vomiting (grade II) } \\
\text { Alopecia (grade I) }\end{array}$ \\
\hline
\end{tabular}

Notes: All drugs were used intravenously if not mentioned otherwise. Side effects were graded according to the CTCAEv5.0 standards. 


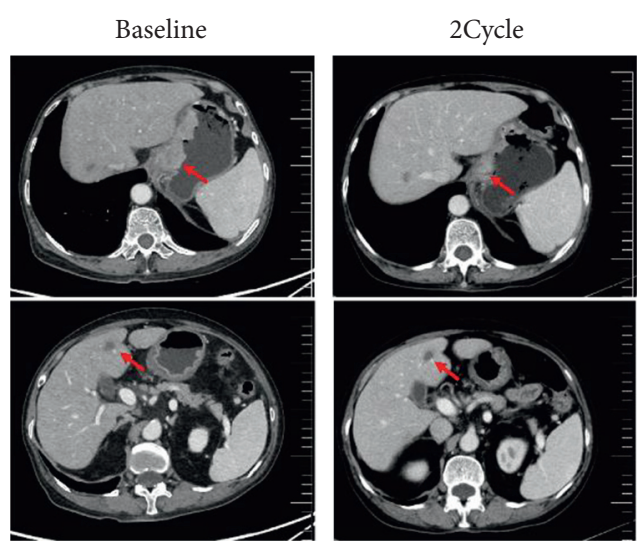

Patient\#1

(a)
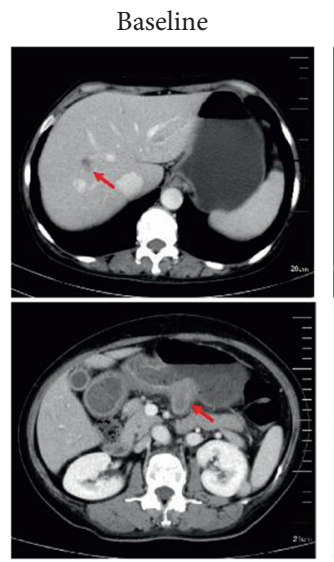

Patient\#3

(c)

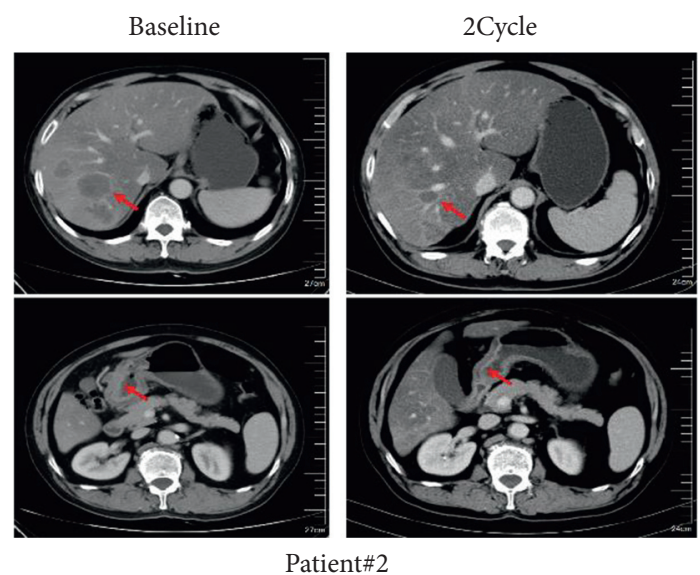

(b)
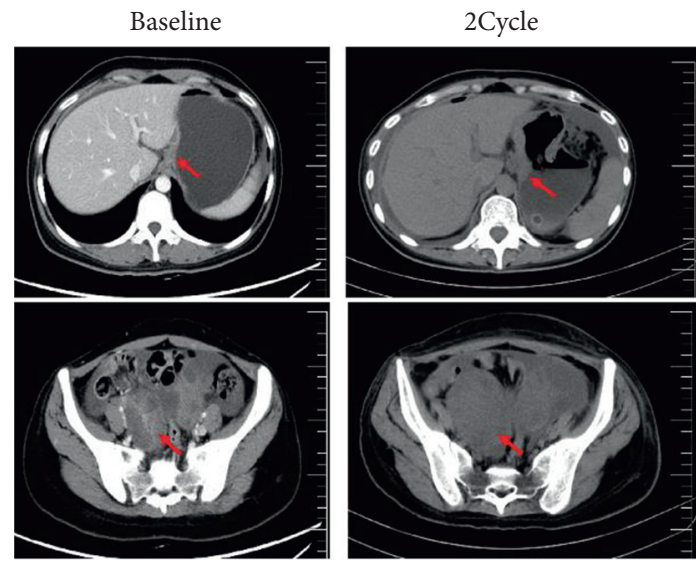

Patient\#4

(d)

FIgURE 4: The clinical efficacy of drugs used in the patients based on the drug sensitivity results of the MiniPDX model. The red arrow indicates the location of the target lesion. CT scans were performed at the baseline and subsequent treatment cycles. Radiographic evidence of the four patients who achieved PR (patient \#1, patient \#2) or PD (patient \#3, patient \#4) after treatment was shown.

Therefore, we can quickly adjust new treatment plans for patients based on the results of drug sensitivity test.

The drug sensitivity experiment technology of the MiniPDX model has been validated in the clinical research of colorectal cancer, hepatocellular carcinoma, gallbladder cancer, ovarian cancer, duodenal cancer, nonsmall cell lung cancer, small cell lung cancer, and other cancer types [19-21, 32-36].

In Li's research of metastatic colorectal cancer, the researchers used three kinase inhibitors (afatinib, gefitinib, and regorafenib) in 31 MiniPDX drug sensitivity testing. The study revealed that the primary tumor and metastases showed a different sensitivity to the same drug even in the same patient [32].

In Zhan's study of gallbladder cancer, the MiniPDX model was established using freshly resected primary lesions from 12 patients with gallbladder to examine the sensitivity of five of the most commonly used chemotherapeutic agents, namely, gemcitabine, oxaliplatin, 5-fluorouracil, nanoparticle albumin-bound (nab) paclitaxel, and irinotecan. The results were used to guide the selection of chemotherapeutic agents for adjunct treatment after the surgery. The Kaplan-Meier method was used to compare the overall survival (OS) and disease-free survival (DFS) of 12 patients in the test group and 45 patients in the control group who received conventional chemotherapy with gemcitabine and oxaliplatin. The result showed that patients in the MiniPDXguided chemotherapy group had significantly longer median OS (18.6 months; 95\% CI 15.9-21.3 months) than patients in the conventional chemotherapy group (13.9 months; 95\% $\mathrm{CI}=11.7-16.2 \quad$ months $) \quad(P=0.030 ; \quad \mathrm{HR}=3.18 ; \quad 95 \%$ $\mathrm{CI}=1.47-6.91)$. Patients in the MiniPDX-guided chemotherapy group also had significantly longer median DFS (17.6 months; 95\% CI $=14.5-20.6$ months) than patients in the conventional chemotherapy group (12.0 months; $95 \%$ $\mathrm{CI}=9.7-14.4 \quad$ months $) \quad(P=0.014 ; \quad \mathrm{HR}=3.37 ; \quad 95 \%$ $\mathrm{CI}=1.67-6.79)[19]$.

The above studies have demonstrated that MiniPDX can perform drug sensitivity testing for different treatment options on different cancers, and patients can benefit from this technology. However, there was no study on gastric cancer using the MiniPDX model. 
There were difficulties in the study. The first one is the location of the tissue biopsy. Some patients have liver metastases, and some have pelvic metastases. After the previous treatment, some of the primary tumors increased, and some of the metastases increased. Because of the difficulty of biopsy from metastases, we only collected tissues of primary gastric cancer. This may not be able to fully reflect the status of all tumors in the patients. The second one is how to evaluate immunotherapy. Immunotherapy has been approved for both the first-line and the third-line treatments of advanced gastric cancer. More and more patients choose a combination of chemotherapy and immunotherapy in the first-line treatment. There are no clinical research data for the maintenance and crossline treatment of immunotherapy in advanced gastric cancer. The MiniPDX model mice are immune system deficient, so only chemotherapy drugs and antiangiogenesis targeted drugs can be chosen as candidates. We often used combined immunotherapy on the basis of chemotherapy. However, whether such a combination is the best choice requires more clinical studies to confirm. The third challenge is how to discuss treatment options with patients. These four patients chose different treatment schemes based on the results of the drug sensitivity experiment of the MiniPDX model. Two patients were evaluated for efficacy as PR, and the other two patients were evaluated for efficacy as PD, which was consistent with the results of the MiniPDX model. Previous studies have shown that if the tumor suppression rate exceeds $55 \%$, the chosen option is most likely to be ineffective. This brought us a lot of confusion. Shall we tell patients to give up treatment? Or continue to have a try? Is palliative treatment a better choice at this time? In addition, due to the limited research funding, our study was limited by a small sample size and a lack of the control group with patients received conventional chemotherapy. At the same time, we also failed to follow up with those 4 subjects to obtain further data such as OS and PFS. It is hoped that funding problems can be solved in our future research. A large randomized controlled clinical trial of MiniPDX technology applied to the drug sensitivity testing for advanced gastric cancer will be carried out.

Although the MiniPDX model has many advantages, it inevitably has some limitations. (1) The MiniPDX model is nonrenewable, requiring a continuous supply of fresh tumor tissue. (2) The usage and cycle of drugs in mice are different from those in humans. When the same drug is used in different cycles (for example, weekly albumin-bound paclitaxel vs. albumin-bound paclitaxel every 3 weeks), the MiniPDX model cannot simulate the actual administration and effect, because the drugs in the MiniPDX model are all used within 7 days. And, (3) the isolation of tumor tissue into single-cell suspension destroys the structure between cells, which may have a certain impact on the tumor microenvironment. Despite these shortcomings, the MiniPDX model still has outstanding advantages in the existing preclinical research models due to its short time-consuming, low cost, and ability to predict the drug treatment efficacy of patients. Especially when it is complementary to other platforms, the MiniPDX model can become a new platform in precision treatment research.

\section{Conclusions}

Our study demonstrated that the MiniPDX is a fast and effective screening model for antitumor drugs. It has particular significance for guiding the choice of drugs for patients with advanced gastric cancer. Recently, the integration of high-throughput sequencing and various indirect in vivo models has contribution to the rapid development of precision therapy. In the future, new and more efficient and comprehensive drug screening models need to be developed to help clinicians for rational use of drugs.

\section{Data Availability}

No data were used to support this study.

\section{Conflicts of Interest}

The authors declare that the research was conducted in the absence of any commercial or financial relationships that could be construed as potential conflicts of interest.

\section{Authors' Contributions}

Jianzheng Wang, Wei Yang, Xiaobing Chen, and Jinxi Huang treated the patients. Hui Wang, Qiwen Bai, and Zhentao Yao were responsible for taking biopsy tissue under endoscopy. Jianzheng Wang, Huifang Lv, Caiyun Nie, Beibei Chen, and Weifeng Xu collected the data. Jianzheng Wang, Hongle Li, Qingli Li, and Shuiping Tu analyzed the data and wrote the original draft. All authors contributed to the article and approved the submitted version. Jianzheng Wang and Jinxi Huang contributed equally to this work.

\section{Acknowledgments}

This work was financially supported by the Science and Technique Foundation of Henan Province (no. 202102310121 for J.-Z. W), the Medical Science and Technology Co-construction Project of Henan Province (no. LHGJ20200167), the 1000 Talents Program of Central plains (no. 204200510023 for X.-B. C), and the Sate Key Laboratory of Esophageal Cancer Prevention \& Treatment (no. Z2020000X for X.-B. C).

\section{References}

[1] J. Machlowska, J. Baj, M. Sitarz, R. Maciejewski, and R. Sitarz, "Gastric cancer: epidemiology, risk factors, classification, genomic characteristics and treatment strategies," International Journal of Molecular Sciences, vol. 21, no. 11, 2020.

[2] X. Y. Zhang and P. Y. Zhang, "Gastric cancer: somatic genetics as a guide to therapy," Journal of Medical Genetics, vol. 54, no. 5, pp. 305-312, 2017.

[3] F. H. Wang, X. T. Zhang, Y. F. Li et al., "The Chinese society of clinical oncology (CSCO): clinical guidelines for the diagnosis and treatment of gastric cancer, 2021," Cancer Communications, vol. 41, no. 8, pp. 747-795, 2021.

[4] Y. J. Bang, E. Van Cutsem, A. Feyereislova et al., "Trastuzumab in combination with chemotherapy versus chemotherapy alone for treatment of HER2-positive advanced 
gastric or gastro-oesophageal junction cancer (ToGA): a phase 3, open-label, randomised controlled trial," The Lancet, vol. 376, no. 9742, pp. 687-697, 2010.

[5] J. Tabernero, P. M. Hoff, L. Shen et al., "Pertuzumab plus trastuzumab and chemotherapy for HER2-positive metastatic gastric or gastro-oesophageal junction cancer (JACOB): final analysis of a double-blind, randomised, placebo-controlled phase 3 study," The Lancet Oncology, vol. 19, no. 10, pp. 1372-1384, 2018.

[6] J. R. Hecht, Y. J. Bang, S. K. Qin et al., "Lapatinib in combination with capecitabine plus oxaliplatin in human epidermal growth factor receptor 2-positive advanced or metastatic gastric, esophageal, or gastroesophageal adenocarcinoma: TRIO-013/LOGiC-a randomized phase III trial," Journal of Clinical Oncology, vol. 34, no. 5, pp. 443-451, 2016.

[7] M. Moehler, "LBA6_PR Nivolumab (nivo) plus chemotherapy (chemo) versus chemo as first-line (1L) treatment for advanced gastric cancer/gastroesophageal junction cancer (GC/GEJC)/esophageal adenocarcinoma (EAC): First results of the CheckMate 649 study-science direct," Annals of Oncology, vol. 31, 2020.

[8] L.-T. Chen, Y.-K. Kang, M. Tanimoto, and N. Boku, "777TiPATTRACTION-04 (ONO-4538-37): a randomized, multicenter, phase $2 / 3$ study of nivolumab (Nivo) plus chemotherapy in patients (Pts) with previously untreated advanced or recurrent gastric $(\mathrm{G})$ or gastroesophageal junction (GEJ) cancer," Annals of Oncology, vol. 28, no. suppl_5, 2017.

[9] H. C. Chung, Y. J. Bang, C. Fuchs et al., "First-line pembrolizumab/placebo plus trastuzumab and chemotherapy in HER2-positive advanced gastric cancer: KEYNOTE-811," Future Oncology, vol. 17, no. 5, pp. 491-501, 2021.

[10] X. Z. Wang, Z. Y. Zeng, X. Ye, J. Sun, Z. M. Zhang, and W. M. Kang, "Interpretation of the development of neoadjuvant therapy for gastric cancer based on the vicissitudes of the NCCN guidelines," World Journal of Gastrointestinal Oncology, vol. 12, no. 1, pp. 37-53, 2020.

[11] M. C. Hernandez, J. R. Bergquist, J. L. Leiting et al., "Patientderived xenografts can be reliably generated from patient clinical biopsy specimens," Journal of Gastrointestinal Surgery, vol. 23, no. 4, pp. 818-824, 2019.

[12] E. Rosfjord, J. Lucas, G. Li, and H. P. Gerber, "Advances in patient-derived tumor xenografts: from target identification to predicting clinical response rates in oncology," Biochemical Pharmacology, vol. 91, no. 2, pp. 135-143, 2014.

[13] H. Gao, J. M. Korn, S. Ferretti et al., "High-throughput screening using patient-derived tumor xenografts to predict clinical trial drug response," Nature Medicine, vol. 21, no. 11, pp. 1318-1325, 2015.

[14] J. J. Tentler, A. Choon Tan, C. D. Weekes et al., "Patientderived tumour xenografts as models for oncology drug development," Nature Reviews. Clinical Oncology, vol. 9, no. 6, pp. 338-350, 2018.

[15] P. Jiménez Fonseca, A. Carmona-Bayonas, R. Hernández et al., "Lauren subtypes of advanced gastric cancer influence survival and response to chemotherapy: real-world data from the AGAMENON national cancer registry," British Journal of Cancer, vol. 117, no. 6, pp. 775-782, 2017.

[16] T. Namikawa, N. Ishida, S. Tsuda et al., "Prognostic significance of serum alkaline phosphatase and lactate dehydrogenase levels in patients with unresectable advanced gastric cancer," Gastric Cancer, vol. 22, no. 4, pp. 684-691, 2019.

[17] S. Iizumi, A. Takashima, K. Sakamaki, S. Morita, and N. Boku, "Survival impact of post-progression chemotherapy in advanced gastric cancer: systematic review and meta-analysis,"
Cancer Chemotherapy and Pharmacology, vol. 81, no. 6, pp. 981-989, 2018.

[18] F. Zhang, W. Wang, Y. Long et al., "Characterization of drug responses of mini patient-derived xenografts in mice for predicting cancer patient clinical therapeutic response," Cancer Communications, vol. 38, no. 1, p. 60, 2018.

[19] M. Zhan, R. M. Yang, H. Wang et al., "Guided chemotherapy based on patient-derived mini-xenograft models improves survival of gallbladder carcinoma patients," Cancer Communications, vol. 38, no. 1, p. 48, 2018.

[20] L. Yang, Z. Yuan, Y. Zhang et al., "MiniPDX-guided postoperative anticancer treatment can effectively prolong the survival of patients with hepatocellular carcinoma," Cancer Chemotherapy and Pharmacology, vol. 87, no. 1, pp. 125-134, 2021.

[21] Y. Huang, J. Xu, K. Li, J. Wang, Y. Dai, and Y. Kang, “A novel, personalized drug-screening system for platinum-resistant ovarian cancer patients: a preliminary clinical report," Cancer Management and Research, vol. 13, pp. 2849-2867, 2021.

[22] K. Shitara, A. Takashima, K. Fujitani et al., "Nab-paclitaxel versus solvent-based paclitaxel in patients with previously treated advanced gastric cancer (ABSOLUTE): an open-label, randomised, non-inferiority, phase 3 trial," The Lancet Gastroenterology \& Hepatology, vol. 2, no. 4, pp. 277-287, 2017.

[23] A. Digklia and A. D. Wagner, "Advanced gastric cancer: current treatment landscape and future perspectives," World Journal of Gastroenterology, vol. 22, no. 8, pp. 2403-2414, 2016.

[24] N. McGranahan and C. Swanton, "Clonal heterogeneity and tumor evolution: past, present, and the future," Cell, vol. 168, no. 4, pp. 613-628, 2017.

[25] G. Khandelwal, M. R. Girotti, C. Smowton et al., "Nextgeneration sequencing analysis and algorithms for PDX and CDX models," Molecular Cancer Research, vol. 15, no. 8, pp. 1012-1016, 2017.

[26] Y. Koga and A. Ochiai, "Systematic review of patient-derived xenograft models for preclinical studies of anti-cancer drugs in solid tumors," Cells, vol. 8, no. 5, 2019.

[27] J. G. Clohessy and P. P. Pandolfi, "Mouse hospital and coclinical trial project-from bench to bedside," Nature Reviews Clinical Oncology, vol. 12, no. 8, pp. 491-498, 2015.

[28] J. G. Clohessy and P. P. Pandolfi, "The mouse hospital and its integration in ultra-precision approaches to cancer care," Frontiers in Oncology, vol. 8, p. 340, 2018.

[29] X. Zhang, S. Claerhout, A. Prat et al., "A renewable tissue resource of phenotypically stable, biologically and ethnically diverse, patient-derived human breast cancer xenograft models," Cancer Research, vol. 73, no. 15, pp. 4885-4897, 2013.

[30] E. L. Stewart, C. Mascaux, N. A. Pham et al., "Clinical utility of patient-derived xenografts to determine biomarkers of prognosis and map resistance pathways in EGFR-mutant lung adenocarcinoma," Journal of Clinical Oncology, vol. 33, no. 22, pp. 2472-2480, 2015.

[31] R. Vargas, P. Gopal, G. B. Kuzmishin et al., "Case study: patient-derived clear cell adenocarcinoma xenograft model longitudinally predicts treatment response," Npj Precision Oncology, vol. 2, no. 1, p. 14, 2018.

[32] C. Li, Y. D. Sun, G. Y. Yu et al., "Integrated omics of metastatic colorectal cancer," Cancer Cell, vol. 38, no. 5, pp. 734-747, 2020.

[33] P. Zhao, H. Chen, D. Wen, S. Mou, F. Zhang, and S. Zheng, "Personalized treatment based on mini patient-derived xenografts and WES/RNA sequencing in a patient with 
metastatic duodenal adenocarcinoma," Cancer Communications, vol. 38, no. 1, p. 54, 2018.

[34] Y. Xu, F. Zhang, X. Pan et al., "Xenograft tumors derived from malignant pleural effusion of the patients with non-small-cell lung cancer as models to explore drug resistance," Cancer Communications, vol. 38, no. 1, p. 19, 2018.

[35] X. Wang, Y. Sun, Y. Xu et al., "Mini-patient-derived xenograft assay based on microfluidic technology promises to be an effective tool for screening individualized chemotherapy regimens for advanced non-small cell lung cancer," Cell Biology International, vol. 45, no. 9, pp. 1887-1896, 2021.

[36] J. Wang, T. Sun, Z. Meng et al., "XPO1 inhibition synergizes with PARP1 inhibition in small cell lung cancer by targeting nuclear transport of FOXO3a," Cancer Letters, vol. 503, pp. 197-212, 2021. 